\title{
ASPECTS OF SOIL MOISTURE AND IRRIGATION
}

By D. S. RICKARD, Chemist, Department of Agriculture's Winchmore Irrigation Research Station.

The growth of all plants depends on a supply of water which is normally obtained through their roots from the soil. Plants use enormous quanities of water during their growing period, and although some of this water is used to carry soluble food into the roots and some is used to assist with carbohydrate production, the amounts used in these ways are small compared with the total consumption. For where a large proportion of other nutrients absorbed_by the-plant is retained,-a_continuous_flow_of water from the soil into the plant roots and out through the leaves must be maintained.

Irrigation is a method of soil management designed to maintain the water content of the soil at a level adequate to meet the demand of the growing plant. Without considering at this stage problems of farming economics and management, there are two main questions to be answered:

(i) When should water be applied?

(ii) How much water is required?

To attempt to answer these deceptively easy questions, some of the principles underlying the behaviour of water in the soil will have to be described. Because of the complexity of the field this will have to be confined to fairly general statements, and it should be kept in mind that a general statement which might be true for one soil can be misleading, or incorrect, for another. Our particular interest in the following is in soil moisture conditions under permanent or semi-permanent pasture.

\section{General}

Soil is a porous medium and the pore space consists of all the space occupied by air and water. The total pore space may make up from 30 to 50 per cent. of the volume of a topsoil, and the character of these pore spaces-their size and distributiondecides to a considerable extent the behaviour of water in the soil. They range in size from the very minute pores in the soil crumbs to the relatively larger holes and cracks in between. Different soils have different characteristics in this respect; a 
greater proportion of the pores in sands are large, while in clay soils most of the pores are very small. Water which enters the soil either remains in these pores or percolates through them to lower depths. Water retention and movement, therefore, are the two important phases of soil moisture to be discussed.

\section{W ater $R$ etention}

If all the pore spaces in a soil were filled with water, that is, if there were no air in the soil at all, the soil would be saturated or waterlogged. This occurs on many soils for a short time after heavy rain or irrigation and persists longer on badly drained or very heavy soils. Normally, however, a great deal of this water will drain away, leaving the soil in a state of field capacity. Field capacity is the condition of the soil two or three days after heavy rain or irrigation when downward drainage has, for all practical purposes, stopped. Obviously it can exist on only well-drained soils, and for these it represents the upper limit of soil moisture availability. The soil moisture content at field capacity depends mainly on the type of soil; it is fairly low for sandy soils, medium for silt loams, and high for clay soils.

Usually, soil moisture levels in a soil are expressed as the percentage by weight of water in a soil sample. A more useful unit of moisture content, however, is the equivalent depth of water over the area considered. This brings the moisture content of the soil into the same units as rainfall (or water applied by spray irrigation) and allows us to express the moisture in a particlar depth of soil in inches of water. That is, at field capacity, a soil may be said to hold $2 \mathrm{in}$ : or $2 \frac{1}{2} \mathrm{in}$. or $3 \mathrm{in}$. of water per foot depth of soil.

A soil at field capacity supporting a vigorous pasture will be subjected to a drying-out process. This is due to evaporation from the surface of the soil and transpiration through the leaves of the pasture plants, the combined effect of which is known as evapotranspiration. If the pasture provides a good cover, the amount of moisture lost directly from the soil will not be great, and most of the drying will be due to transpiration losses. The rate of transpiration, and hence the rate at which the soil will dry, will be largely controlled by the weather, particularly temperature, sunshine, wind, and humidity. An actively growing pasture will transpire an enormous quantity of water over its growing period, probably something over 2000 tons per acre per season. Over a period of hot, rainless, sunny days, the moisture content of the soil will fall at a rate equivalent to a loss of 10 to 20 points of rain per day. As the soil dries, the remaining water is more and more tightly held by the soil until a point is reached when the 
plant roots cannot extract any more water from the soil. The pasture will suffer from a lack of water and will wilt and brown off. By then, the soil will have dried to the lower limit of moisture availability known as the permanent wilting point. This is a fairly constant moisture level for any particular soil type, and the pasture will not grow when the soil is below wilting point. Although, for the sake of convenience, we refer to it as a "point", wilting takes place over a range of soil moisture, and growth is likely to be adversely affected before actual wilting takes place. A representation of the drying of a soil is shown in Fig. 1.

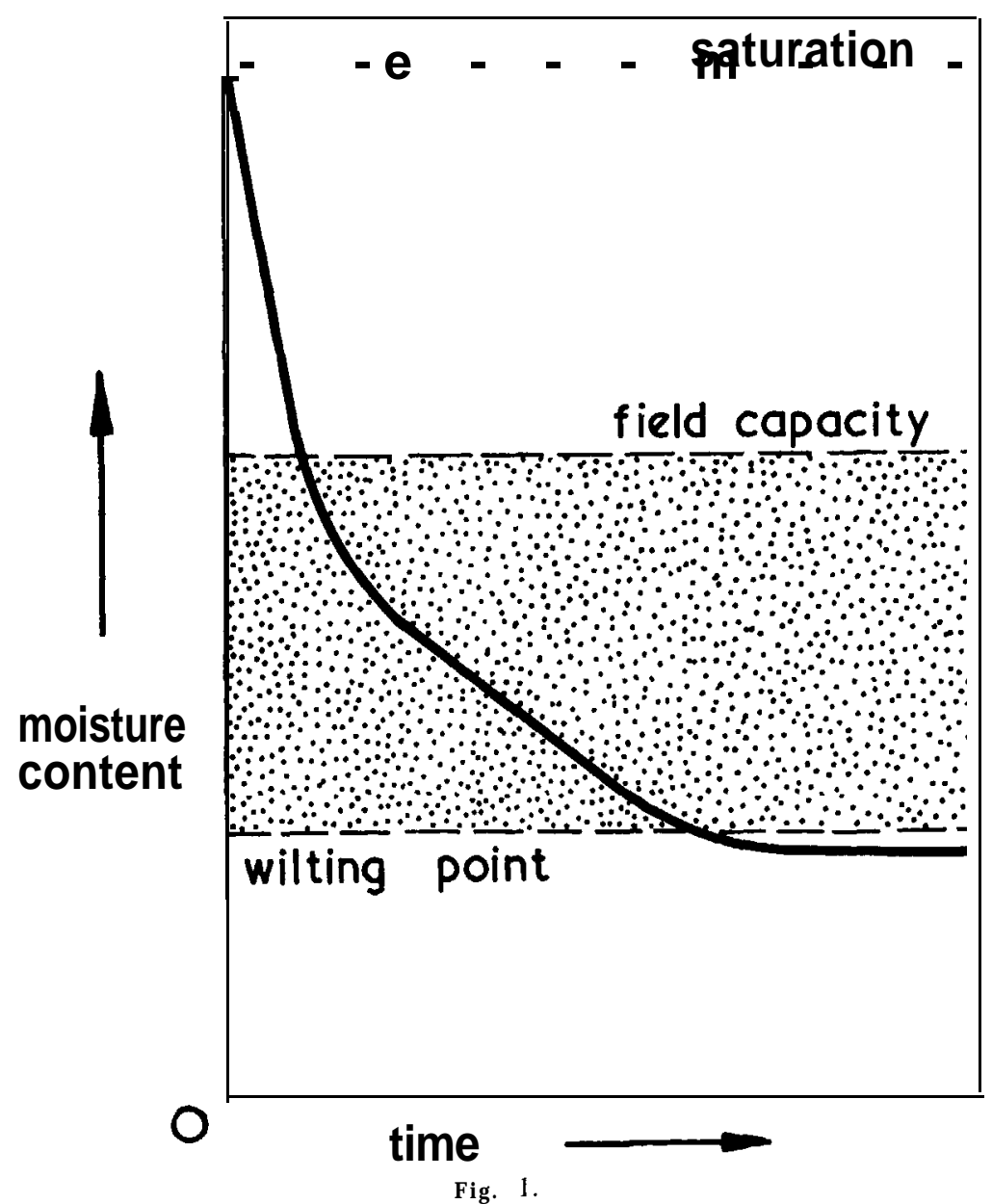

These two points on the soil-water scale are of great importance. A measure of field capacity will tell us how much water the soil 
can hold; a measure of wilting point will tell us at what point the amount of water in the soil is insufficient for pasture growth. Moisture held in the soil between field capacity and wilting point is available moisture; available, that is, for plant growth. The general relationship between field capacity and wilting point for a range of soil types is shown in Fig. 2. Although the value of field capacity increases as the clay content increases, the level at which permanent wilting occurs also increases. A high field capacity for a soil does not therefore necessarily mean a greater amount of available moisture.

inches of water

per foot of soil.

4

3

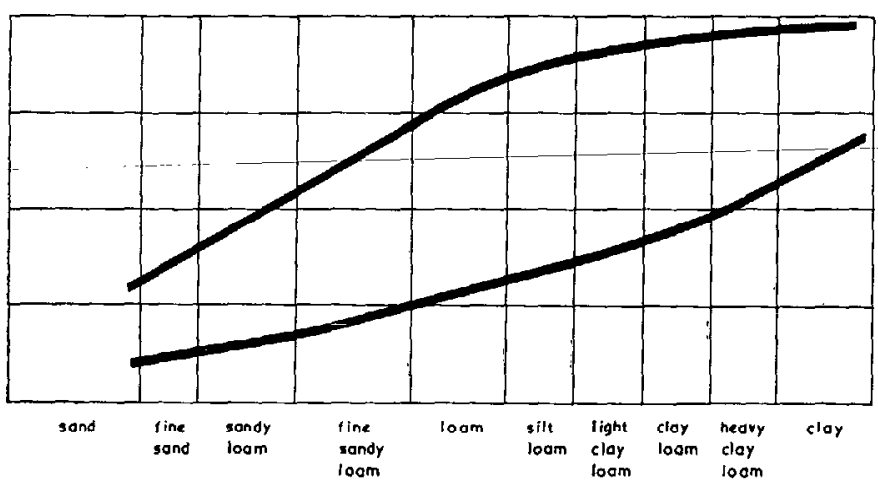

Fig. 2.

(Adapted from graph on p. 120 U.S.D.A. Yearbook of Agriculture 1955.)

If we return to our original definition of irrigation as a means of keeping the moisture in the soil at an adequate level for plant growth, it is apparent that water should be applied either before, or possibly at, wilting point. To allow it to dry out beyond this will seriously retard growth. This brings us back to one of our original questions: When should water be applied? The answer is not simple, and involves such things as management, stock requirements, water availability, and capacities of spray plants. But if I were asked this question, the answer would probably be: When $\frac{1}{2}$ to $\frac{3}{4}$ of the available moisture in the soil had been used up. This means that on a soil holding 3in. of water at field capacity, 2in. of which is available, irrigation should be applied when I to $1 \frac{1}{2}$ in. had been used. The question of how this can be measured or estimated cannot be covered in the time available. Methods involving the calculation of transpiration losses from meteorological data that are extensively used overseas are being 
investigated in New Zealand and seem to offer the best solution to this problem.

The answer to our second question-how much water is required?-is comparatively simple once we can answer the first. Just enough water should be added to bring the soil in the root zone back to field capacity. This avoids over-watering-and possible waterlogging, waste of water, and leaching-and also prevents under-watering when the full benefit of the irrigation would not be obtained.

\section{Movement of Water}

For saturated soils-that is, soils above field capacity-the mechanics of water movement are reasonably well known and obey certain laws. Unfortunately, we are not, in general, interested in saturated soils, and the problem of water movement in soils with a moisture content below field capacity is rather more difficult. It is, however, a fact that water movement in such soils is very slow, When water is added to a soil, the wet zone will spread downward and sideways fairly rapidly until a volume of soil has been raised to field capacity. The addition of more water to the soil will extend this zone of field capacity downwards. If no more water is added, there will remain an area of soil at field capacity in contact with soil at the original moisture level. The rate of movement from the moist to the dry soil will be slow, too slow to supply water at a sufficient rate to plant roots in the dry soil.

The extremely slow rate of movement means that water tends to stay where it is in a soil and this has two important practical implications. The first is that it is not possible to wet soil to any predetermined level lower than field capacity. That is, if we have 12in. depth of soil at wilting point, and enough water is applied to bring all the soil to an average of half-way between field capacity and wilting point, we will end up with the top 6in. of soil at field capacity and the remaining 6in. still at wilting point. The addition of more water to the surface will result in a greater depth being wet to field capacity. The other implication of the slow rate of water movement in moist soils is that plant roots must seek out moisture in the soil and not rely on moisture moving through the soil to them. In irrigating we must apply water to the root zone, to where it will do the most good.

There is another way in which water may move through the soil; as a vapour. In a soil with a uniform temperature throughout, the amount which moves in this way is negligible. But if there are considerable differences in temperature throughout the soil, more water may move in this way than as a liquid, particularly when 
the soil is near wilting point. This may result in upward diffusion of water vapour to the surface of the soil and consequent loss by evaporation.

\section{Summary}

We can now summarise the important points in relation to soil moisture and irrigation.

Soil contains crumbs or compound particles between which there are pore spaces filled with air. The crumbs themselves contain very minute pores, much smaller than the spaces between the crumbs. Irrigation water fills the pores in the soil crumbs at the top layer of soil, and the excess water passes through the larger pores to be absorbed by the soil crumbs below. If only a light amount of water is applied, only the top few inches will be wet, and the remaining soil will be relatively unaffected. If, however, more water is applied, this "front" of wetted soil moves downward. When there is no further tendency for moisture to move down the soil profile, the soil is at field capacity. From then, the greatest loss of moisture from the soil takes place by transpiration through the plant leaves. During an exceptionally hot day, the equivalent of 4500 gallons or 20 tons of water per acre per day may be removed in this way. Under rainless conditions all the available moisture in the soil may be used up and the pasture show signs of moisture deficit. If we do not wish pasture production to suffer through lack of water, irrigation must be applied before this happens. Water is required before the wilting point is reached, and enough should be applied to raise the whole root zone to field capacity.

\section{DISCUSSION}

Q. What is the maximum quantity of water containing a high concentration of nutrients which can be irrigated on to a fairly heavy soil without depressing pasture growth too much?

A. This would depend entirely on the soil type. On the soil at Winchmore, for example, very large quantities of water could be applied without adversely affecting pasture growth. On another soil type, water-logging may occur which would badly affect the pasture.

Q. With sub-soil irrigation how high do you have to raise the watertable to get optimum growth?

A. This again depends upon soil type, but the root zone should be raised to field capacity.

Professor Hudson: The real problem of lifting water from the watertable is one of soil texture and here the organic matter content plays an important role. Water will rise in a front according to the capilliary fringe which is determined by the type of soil. 
Q. Has the speaker any views on subsoiling as a micans of increasing the water holding capacity of the soil?

A. No.

Professor Hudson: Subsoiling is today getting a considerable degree of prominence due to heavy machinery being readily available. On heavy clay soils under high rainfall conditions subsoiling can result in increased wetness.

Q. On soils under a vigorous pasture would not worm holes and cracks affect the picture of permeability as presented?

A. The concept of water movement through the soil as given in the paper is, of course, over-simplified. Irregularities in the soil-patches of organic matter, stones, etc., will have an effect on the penetration of water. In the main, however, the general picture will be very much as given.

Q. On soils under a vigorous pasture what effect do worms and other forms of biological activity have on the soil's permeability?

A. The concept of soil moisture movement as given in the paper is over simplified and factors such as earthworm activity would not make the rate of percolation irregular.

Professor Hudson: Mr G. Hopewell when at Massey College did some work in this connection and he might like to discuss the matter.

Mr Hopewell: We watered a small plot on Manawatu silt loam which had an impervious subsoil $18 \mathrm{in}$. to $21 \mathrm{in}$. down The water applied went down cracks in the topsoil to the impervious layer where it commenced to build up. It was followed by the normal wetting front. This meant that there was a stage where there was a uniform wetting front advancing upon a dryer soil area and a zone of free water accumulating at the impervious layer. 